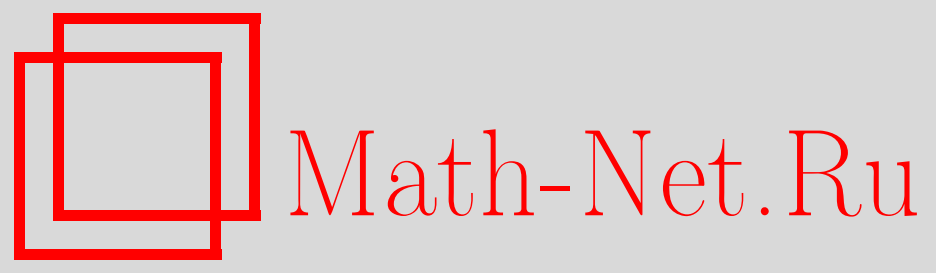

Й. Брюнинг, С. Ю. Доброхотов, М. И. Кацнельсон, Д. С. Миненков, Квазиклассические асимптотики и плотность состояний для двумерных центральносимметричных уравнений Шредингера и Дирака в задачах туннельной микроскопии, ТМФ, 2016, том 186, номер 3, 386-400

DOI: https://doi.org/10.4213/tmf8987

Использование Общероссийского математического портала Math-Net.Ru подразумевает, что вы прочитали и согласны с пользовательским соглашением http://www . mathnet.ru/rus/agreement

Параметры загрузки:

IP : 54.81 .137 .203

26 апреля 2023 г., $17: 50: 20$

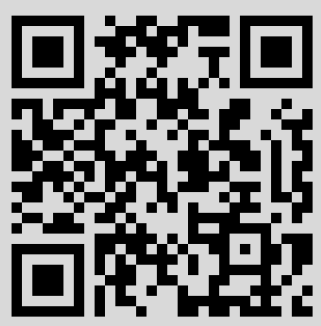


Й. Брюнинг ${ }^{*}$, С. Ю. Доброхотов ${ }^{\dagger \ddagger}$, М. И. Кацнельсон ${ }^{\S}$, Д. С. Миненков ${ }^{\dagger \ddagger}$

\title{
КВАЗИКЛАССИЧЕСКИЕ АСИМПТОТИКИ И ПЛОТНОСТЬ СОСТОЯНИЙ ДЛЯ ДВУМЕРНЫХ ЦЕНТРАЛЬНО-СИММЕТРИЧНЫХ УРАВНЕНИЙ ШРЕДИНГЕРА И ДИРАКА В ЗАДАЧАХ ТУННЕЛЬНОЙ МИКРОСКОПИИ
}

\begin{abstract}
Рассматриваются двумерные стационарные уравнения Шредингера и Дирака с центрально-симметричным потенциалом, моделирующим заряженную иглу сканирующего туннельного микроскопа. На основе асимптотик для обобщенных собственных функций вычислена плотность состояний под иглой, связанная с измерениями с помощью микроскопа. Показано, что в случае уравнения Дирака игла микроскопа искажает измеряемую плотность состояний при всех энергиях.
\end{abstract}

Ключевые слова: радиально-симметричные двумерные операторы Шредингера и Дирака, обобщенные собственные функции, квазиклассическое приближение, плотность состояний, туннельная микроскопия.

DOI: $10.4213 / \operatorname{tmf} 8987$

Настоящая работа выполнена при поддержке грантов DFG-RAS (№ 2436 RUS 113/990/0-1), Германия, РФФИ (№ 12-01-31196) и Государственной программы поддержки ведущих университетов (Постановление 211 Правительства РФ, контракт № 02.A03.21.006).

${ }^{*}$ Humboldt University, Berlin, Germany. E-mail: bruening@mathematik.hu-berlin.de

${ }^{\dagger}$ Институт проблем механики им. А. Ю. Ишлинского РАН, Москва, Россия. E-mail: dobr@ipmnet.ru, minenkov.ds@gmail.com

${ }^{\ddagger}$ Московский физико-технический институт, Долгопрудный, Московская обл., Россия

${ }^{\S}$ Institute for Molecules and Materials, Radboud University, Nijmegen, the Netherlands. E-mail: M.Katsnelson@science.ru.nl

ॠ Уральский федеральный университет им. первого Президента России Б. Н. Ельцина, Екатеринбург, Россия 


\section{1. ВВЕДЕНИЕ}

Наше исследование связано со следующим вопросом: насколько игла сканирующего туннельного микроскопа [1] влияет на измеряемые величины? Исследуется влияние потенциала, индуцированного иглой микроскопа, на электронные состояния в кристалле. Мы рассматриваем ситуацию, когда на подложке расположен двумерный кристалл, к которому поднесена игла электронного микроскопа. Между иглой и подложкой задается разность потенциалов $U_{0}$, в результате чего между иглой и кристаллом возникает туннельный ток, который измеряется микроскопом и который пропорционален локальной плотности состояний (а также плотности электронов) в точке кристалла под иглой (см. работы [2], [3] и библиографию в них). В то же время в кристалле возникает потенциал $U(r)$, наведенный иглой микроскопа и искажающий измеряемую плотность состояний. Математическая постановка задачи такова: требуется построить асимптотики для обобщенных собственных функций стационарных уравнений Шредингера и Дирака на плоскости с центрально-симметричным потенциалом $U(r)$ и определить локальную плотность состояний в точке максимума потенциала (т. е. под иглой).

Мы рассматриваем плоскую подложку и предполагаем, что двумерный кристалл "прозрачен" для поля. Используя модель из работы [4] для электростатического поля иглы рядом с плоским экраном, мы моделируем электростатический потенциал между иглой и подложкой, в том числе электростатический потенциал

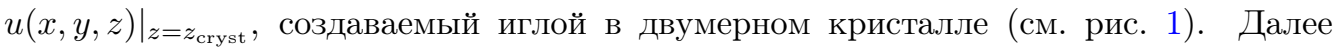
мы рассматриваем двумерное уравнение Шредингера в случае кристалла с квадратной кристаллической решеткой или двумерное уравнение Дирака для кристалла с гексагональной решеткой. В уравнения входит центрально-симметричный потенциал $U(x, y)=\left.q_{e} u(x, y, z)\right|_{z=z_{\text {cryst }}}$, наведенный иглой микроскопа (здесь $q_{e}$ - заряд электрона). Будем считать, что потенциал $U(r)$ монотонно убывает и стремится на бесконечности к нулю. Предполагается, что масса $m(r)$ в уравнении Дирака центрально-симметрична и имеет на бесконечности конечный предел (не обязательно равный нулю), например, можно рассматривать постоянную массу.

После введения характерного масштаба энергии $E_{0}$ и длины $L_{0}$ получаются безразмерные уравнения с малым (квазиклассическим) параметром перед производными. Рассмотрим двумерное стационарное уравнение Шредингера

$$
-h^{2} \Delta \psi-(E-U(r)) \psi=0
$$

или уравнение Дирака

$$
\left(\begin{array}{cc}
m(r) & -i h e^{-i \varphi}\left(\frac{\partial}{\partial r}-\frac{i}{r} \frac{\partial}{\partial \varphi}\right) \\
-i h e^{i \varphi}\left(\frac{\partial}{\partial r}+\frac{i}{r} \frac{\partial}{\partial \varphi}\right) & -m(r)
\end{array}\right) \Psi-(E-U(r)) \Psi=0,
$$

где $x \in \mathbb{R}^{2}, x_{1}=r \cos \varphi, x_{2}=r \sin \varphi$,

$$
\Delta=\frac{\partial^{2}}{\partial r^{2}}+\frac{1}{r} \frac{\partial}{\partial r}+\frac{1}{r^{2}} \frac{\partial^{2}}{\partial \varphi^{2}}
$$

есть оператор Лапласа, $U(r)=U(|x|)$ - потенциал, $m(r)$ - масса, $h \ll 1$ - квазиклассический параметр, возникающий в результате обезразмеривания: $h^{2} \equiv \hbar^{2} /\left(2 m_{e} E_{0} L_{0}^{2}\right)$ 

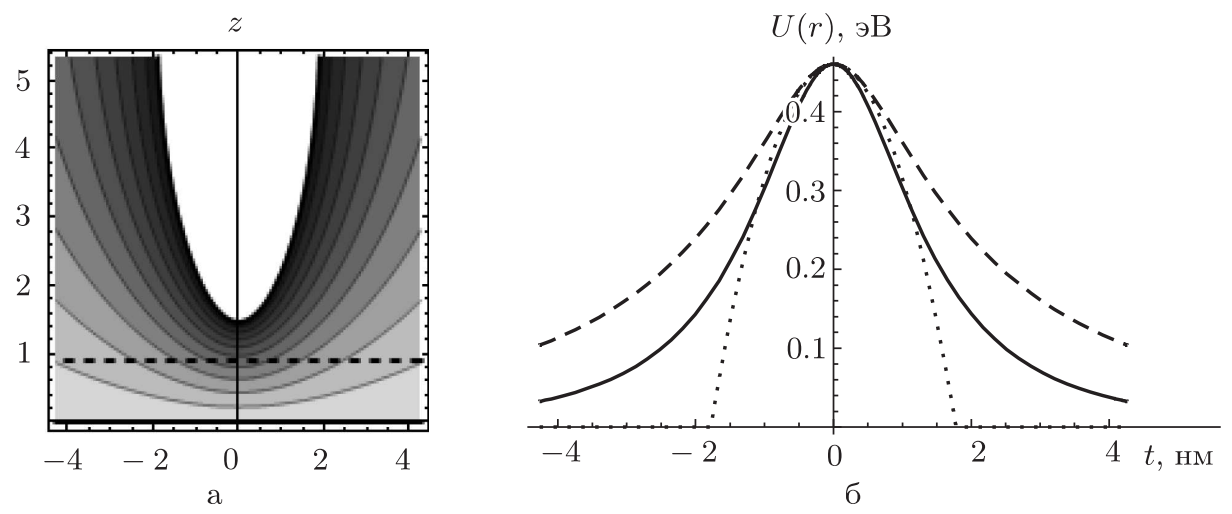

Рис. 1. Эквипотенциальные линии поля иглы микроскопа (а); белая область отмечает моделируемую поверхность иглы микроскопа, сплошная линия $z=0$ - подложку, штриховая линия - рассматриваемый двумерный кристалл. Потенциал в двумерном кристалле (б) для иглы (сплошная линия), точечного заряда (штриховая линия) и модельного парабалического потенциала (пунктир).

для уравнения Шредингера и $h \equiv \hbar v_{\mathrm{F}} /\left(E_{0} L_{0}\right)$ для уравнения Дирака, $\hbar-$ постоянная Планка, $m_{e}$ - масса электрона, $v_{\mathrm{F}}$ - скорость Ферми. При расчетах используются параметры иглы микроскопа из работы [5], индуцированный потенциал изображен на рис. 1.

Для расчета локальной плотности состояний можно использовать два подхода. Один из них заключается в том, что рассматривается задача в ограниченной области $r=\sqrt{x^{2}+y^{2}} \leqslant R$ с граничными условиями при $r=R$ (например, условиями Дирихле $\left.\psi\right|_{r=R}=0$ для уравнения Шредингера) и вычисляется плотность состояний для дискретного спектра $E_{k}$ по формуле [6]

$$
\left.\operatorname{LDOS}\left(E_{k}\right)\right|_{r=0}=\left|\psi_{k}(0)\right|^{2} D\left(E_{k}\right)=\frac{\left|\psi_{k}(0)\right|^{2}}{\Delta E_{k}}, \quad \Delta E_{k} \equiv E_{k+1}-E_{k} .
$$

Другой вариант - рассматривать задачу в неограниченной области $(x, y) \in \mathbb{R}^{2}$, ставить условие нормировки собственных функций непрерывного спектра на дельтафункцию, $\iint_{\mathbb{R}^{2}} \psi_{k} \psi_{k_{1}}^{*} d x=\delta\left(k-k_{1}\right)$, и определять плотность состояний для непрерывного спектра как (см. например, книгу [7])

$$
\left.\operatorname{LDOS}(E)\right|_{r=0} \equiv \int|\psi(0, E(k))|^{2} \delta(E(k)-E) d k=\left.|\psi(0, E)|^{2}\left(\frac{d E(k)}{d k}\right)^{-1}\right|_{E(k)=E} .
$$

Зависимость энергии $E(k)$ от модуля волнового числа $k$ имеет вид $E(k)=h^{2} k^{2}$, $d E(k) / d k=2 h^{2} k=2 h \sqrt{E(k)}$ в случае уравнения Шредингера и вид $E(k)=h k$, $d E(k) / d k=h$ в случае уравнения Дирака.

Как показано в монографии [6], эти подходы эквивалентны для точных собственных функций: в пределе $R \rightarrow \infty$ плотность состояний для дискретного спектра переходит в плотность состояний для непрерывного спектра. В случае конечной области 
можно сформулировать точный математический результат для асимптотик волновых функций: поскольку задача центрально-симметричная и потому эффективно одномерная, асимптотики являются асимптотическими волновыми функциями, т. е. приближают точные решения. Асимптотики имеют вид функций Бесселя [8]. Окончательные формулы зависят от размера области $R$ и довольно громоздкие. В случае бесконечной области окончательные формулы имеют простой наглядный вид, но теорем о том, что представленные формулы являются асимптотиками решения, насколько нам известно, нет. Тем не менее если формально использовать полученные асимптотические формулы для задачи с неограниченной областью, нормировать их на дельта-функцию и найти для них плотность состояний, то ответ для плотности состояний совпадает с пределом асимптотической плотности состояний для ограниченной области при $h=$ const $>0, R \rightarrow \infty$.

Таким образом, ответ для плотности состояний, рассчитанный в случае бесконечной области для "асимптотической волновой функции", имеет простой наглядный вид и представляется физически разумным.

Структура работы следующая. В разделе 2 проводится разделение переменных в центрально-симметричном случае. Систему Дирака в этом случае можно свести к двум уравнениям типа возмущенного уравнения Шредингера с эффективным потенциалом, зависящим от исходного потенциала, энергии и массы (см. монографию [9]). В разделе 3 приводятся формулы для главного члена асимптотики волновой функции в случае бесконечной области и плотность состояний для этих асимптотик. Результаты представлены на рис. 2. В приложении А приведены квазиклассические асимптотики решения (см. рис. 3) и процедура их нормировки на дельта-функцию. В приложении Б выписаны точные решения уравнения Шредингера в случае квадратичного потенциала (см. рис. 4). Для уравнения Дирака точное решение приведено только для уровня энергии $E=U(0)$ и нулевой массы (см. рис. 5$)$.

\section{2. РАЗДЕЛЕНИЕ ПЕРЕМЕННЫХ, РЕДУКЦИЯ СИСТЕМЫ ДИРАКА К СКАЛЯРНЫМ УРАВНЕНИЯМ}

Благодаря симметрии переменные в уравнениях (1.1), (1.2) разделяются, после чего задача становится эффективно одномерной. Уравнение Шредингера (1.1) при подстановке $\psi(x)=e^{i l \varphi} \psi(r), l \in \mathbb{Z}_{+}$, переходит в

$$
-h^{2}\left(\frac{\partial^{2}}{\partial r^{2}}+\frac{1}{r} \frac{\partial}{\partial r}+\frac{l^{2}}{r^{2}}\right) \psi-(E-U(r)) \psi=0
$$

В окрестности $r=0$ решение имеет вид $\psi=c J_{l}\left(\frac{r}{h} \sqrt{E-U_{0}}\right)$, так что если $l \neq 0$, то $\psi(0)=0$. Вклад в плотность состояний в точке $r=0$ дают только собственные функции с нулевым угловым моментом, и достаточно рассмотреть одномерное уравнение

$$
-h^{2}\left(\frac{\partial^{2}}{\partial r^{2}}+\frac{1}{r} \frac{\partial}{\partial r}\right) \psi-n^{2}(r) \psi=0, \quad n^{2}(r)=E-U(r) .
$$


Аналогично, подстановка $\Psi(x)=\left(e^{i l \varphi} \psi_{1}(r), e^{i(l+1) \varphi} \psi_{2}(r)\right)^{\mathrm{T}}$ в систему Дирака дает (см., например, монографию [9])

$$
\begin{gathered}
\psi_{2}=-i h \frac{1}{v_{+}}\left(\frac{\partial}{\partial r}-\frac{1}{r}\right) \psi_{1}, \quad v_{+}(r)=E-U(r)+m(r), \\
-h^{2}\left(\frac{\partial^{2}}{\partial r^{2}}+\frac{1}{r} \frac{\partial}{\partial r}-\frac{l^{2}}{r^{2}}\right) \psi_{1}+h^{2} \frac{v_{+}^{\prime}}{v_{+}}\left(\frac{\partial}{\partial r}-\frac{l}{r}\right) \psi_{1}-\left((E-U)^{2}-m^{2}\right) \psi_{1}=0
\end{gathered}
$$

или, эквивалентно,

$$
\begin{gathered}
\psi_{1}=-i h \frac{1}{v_{-}}\left(\frac{\partial}{\partial r}+\frac{l+1}{r}\right) \psi_{2}, \quad v_{-}(r)=E-U(r)-m(r), \\
-h^{2}\left(\frac{\partial^{2}}{\partial r^{2}}+\frac{1}{r} \frac{\partial}{\partial r}-\frac{(l+1)^{2}}{r^{2}}\right) \psi_{1}+h^{2} \frac{v_{-}^{\prime}}{v_{-}}\left(\frac{\partial}{\partial r}+\frac{l+1}{r}\right) \psi_{2}-\left((E-U)^{2}-m^{2}\right) \psi_{2}=0 .
\end{gathered}
$$

Вклад в плотность состояний в точке $r=0$ дают решения с $l=0$, когда $\psi_{1}(0) \neq 0$, и с $l=-1$, когда $\psi_{2}(0) \neq 0$. Имеем при $l=0$

$$
-h^{2}\left(\frac{\partial^{2}}{\partial r^{2}}+\frac{1}{r} \frac{\partial}{\partial r}\right) \psi_{1}-n_{1}^{2} \psi_{1}+h^{2} \frac{v_{+}^{\prime}}{v_{+}} \frac{\partial}{\partial r} \psi_{1}=0, \quad \psi_{2}=-i h \frac{1}{v_{+}} \psi_{1}^{\prime},
$$

а при $l=-1$

$$
-h^{2}\left(\frac{\partial^{2}}{\partial r^{2}}+\frac{1}{r} \frac{\partial}{\partial r}\right) \psi_{2}-n_{1}^{2} \psi_{2}+h^{2} \frac{v_{-}^{\prime}}{v_{-}} \frac{\partial}{\partial r} \psi_{1}=0, \quad \psi_{1}=-i h \frac{1}{v_{-}} \psi_{2}^{\prime},
$$

где

$$
n_{1}=\sqrt{(E-U)^{2}-m^{2}}, \quad v_{ \pm}(r)=E-U(r) \pm m(r) .
$$

При нулевой массе случай $l=-1$ симметричен относительно перестановки $\psi_{1}$ и $\psi_{2}$, что отражает симметрию относительно компонент псевдоспина.

Мы будем рассматривать уравнения (2.1) и (2.2) в области $r \in \mathbb{R}_{+}$и дополним их условием гладкости при $x=0$ и условием нормировки на дельта-функцию:

$$
\begin{array}{rlrl}
\psi^{\prime}(0) & =0, & \iint_{\mathbb{R}^{2}} \psi_{k} \psi_{k_{1}}^{*} d x & =\delta\left(k-k_{1}\right), \\
\psi_{1}^{\prime}(0) & =0, \quad \iint_{\mathbb{R}^{2}} \Psi_{k} \Psi_{k_{1}}^{*} d x=\iint_{\mathbb{R}^{2}}\left(\psi_{1, k} \psi_{1, k_{1}}^{*}+\psi_{2, k} \psi_{2, k_{1}}^{*}\right) d x=\delta\left(k-k_{1}\right),
\end{array}
$$

где $k$ - модуль волнового вектора, $\psi_{k}=\psi(x ; E(k))$. Для уравнения $(2.3)$ условие гладкости имеет вид $\psi_{2}^{\prime}(0)=0$.

В случае несимметричного потенциала точно разделить переменные и уравнения, как правило, нельзя, и необходимо использовать адиабатический метод [10], [11].

\section{3. ГЛАВНЫЙ ЧЛЕН АСИМПТОТИКИ. ПЛОТНОСТЬ СОстоЯнИй}

Для уравнений (2.1)-(2.3) асимптотическое решение дается каноническим оператором Маслова [12], для которого в данном случае удобно использовать представление, недавно полученное в работе [13]:

$$
\psi(x)=\oint \varphi(x, \theta, h) e^{(i / h) \Phi(x, \theta)} d \theta, \quad \Phi(x, \theta)=T(x \mathbf{n}(\theta)), \quad \mathbf{n}(\theta)=(\cos \theta, \sin \theta),
$$


где эйконал $T(r)=\int_{0}^{r} n(r) d r$ равен $T(r)=\int_{0}^{r} \sqrt{E-U} d r$ для уравнения Шредингера (2.1) и равен $T(r)=\int_{0}^{r} \sqrt{(E-U)^{2}-m^{2}} d r$ для уравнений $(2.2),(2.3)$, к которым сводится уравнение Дирака. Это представление можно использовать и в случае, когда потенциал $U(x)$ не обладает симметрией. В таком представлении можно достаточно просто определить поправки к главному члену асимптотики. С учетом симметрии оно принимает вид интегрального представления функции Бесселя [8]:

$$
\begin{gathered}
\psi(r, h)=\int e^{(i / h) T(r, h) \cos \eta} \varphi(r, h) d \eta=2 \pi \varphi(r, h) J_{0}\left(\frac{1}{h} T(r, h)\right), \\
T(r, h)=T(r)+h T_{1}(r)+\cdots, \quad \varphi(r, h)=\varphi_{0}(r)+h \varphi_{1}(r)+\cdots,
\end{gathered}
$$

где $T_{k}(r), \varphi_{k}(r) \in C^{\infty}\left(\mathbb{R}_{+}\right)$.

Для уравнения Шредингера главный член асимптотики для надбарьерных состояний $E>U(0)$ имеет вид

$$
\psi(r)=\frac{\sqrt[4]{E}}{\sqrt{2 \pi h}} \frac{\sqrt{T(r)}}{\sqrt{r} \sqrt[4]{E-U(r)}} J_{0}\left(\frac{1}{h} T(r)\right)(1+O(h)) .
$$

При $E<U(0)$ в классически запрещенной области (при $r<r_{0}(E)$, где $r_{0}(E)$ - точка поворота, $\left.U\left(r_{0}(E)\right)=E\right)$ главный член асимптотики имеет вид

$\psi(x)=\frac{\sqrt[4]{E}}{\sqrt{2 \pi h}} \exp \left\{-\frac{1}{h} \int_{0}^{r_{0}(E)} \sqrt{U(r)-E} d r\right\} \frac{\sqrt{T_{\mathrm{tun}}(r)}}{\sqrt{r} \sqrt[4]{U(r)-E}} J_{0}\left(\frac{1}{h} T_{\mathrm{tun}}(r)\right)(1+O(h))$,

где

$$
T_{\text {tun }}(r)=\int_{0}^{r}|n(r)| d r=\int_{0}^{r} \sqrt{U(r)-E} d r .
$$

Плотность состояний в нуле для надбарьерных состояний и состояний с классически запрещенной областью соответственно равна

$$
\begin{array}{ll}
\operatorname{LDOS}_{S c h}(E)=\frac{1}{4 \pi h^{2}}(1+O(h)), & E>U(0), \\
\operatorname{LDOS}_{S c h}(E)=\frac{1}{4 \pi h^{2}} \exp \left\{-\frac{2}{h} \int_{0}^{a(E)} \sqrt{U\left(r_{1}\right)-E} d r_{1}\right\}(1+O(h)), & E<U(0) .
\end{array}
$$

Плотность состояний над барьером (первое выражение в (3.4)) совпадает с плотностью состояний при нулевом потенциале. Плотность состояний под барьером является экспоненциально малой. Графики плотности состояний представлены на рис. 2.

В случае уравнения Дирака с нулевой массой эффективный потенциал имеет вид $n_{1}^{2}(r)=|E-U(r)|$, и классически запрещенная область отсутствует. Главный член асимптотики записывается как

$$
\psi_{1}(r)=\frac{1}{\sqrt{2} \sqrt{2 \pi h}} \frac{\sqrt{T(r)}}{\sqrt{r}} J_{0}\left(\frac{1}{h} T(r)\right)(1+O(h)),
$$




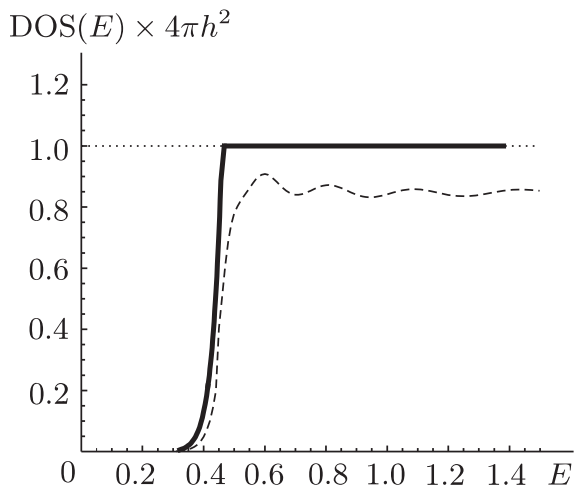

a

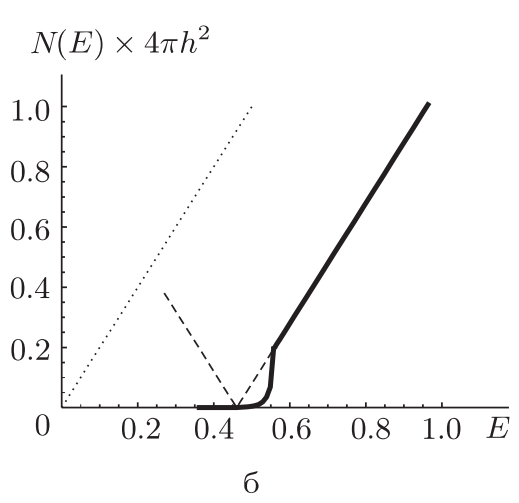

б

Рис. 2. Плотность состояний для уравнения Шредингера (а), соответствующая главному члену асимптотики для потенциала иглы (сплошная линия), точному решению для параболического потенциала (штриховая линия) и точному решению с нулевым потенциалом (пунктир). Плотность состояний для уравнения Дирака (б), соответствующая главному члену асимптотики, для потенциала в случае ненулевой массы (сплошная линия), для потенциала в случае нулевой массы (штриховая линия) и для уравнения без потенциала и массы (пунктир). Максимум наведенного потенциала равен $U(0)=0.46$, масса для уравнения Дирака выбрана равной $m=0.1$. Квазиклассический параметр $h=0.1$ в обоих случаях.

а плотность состояний равна (с учетом симметрии относительно компонент $\psi_{1}, \psi_{2}$ псевдоспина)

$$
\operatorname{LDOS}_{\text {Dir }}(E)=\frac{|E-U(0)|}{2 \pi h^{2}}(1+O(h)) .
$$

Если при этом $E<U(0)$, то можно говорить о плотности “дырочных" состояний. Плотность состояний при нулевом потенциале равна $E / 2 \pi h^{2}$. Наличие потенциала приводит к "сдвигу" этой зависимости: плотность состояний уменьшается на величину $U_{0} / 2 \pi h^{2}$, одинаковую для всех энергий $E>U(0)$ выше потенциального барьера (см. рис. 2).

В случае уравнения Дирака с ненулевой массой эффективный потенциал в уравнениях $(2.2),(2.3)$ равен $n_{1}^{2}(r)=(E-U(r))^{2}-m(r)^{2}$ и может принимать отрицательные значения, т. е. возникает классически запрещенная область. При ненулевой массе также нарушается симметрия относительно компонент псевдоспина.

Рассмотрим несколько режимов.

РЕжим 1: $E>U(0)+m(0)$. В этом случае при всех $r \in \mathbb{R}_{+}$эффективный потенциал положителен и волновая функция является осциллирующей.

РЕжим 2: $U(0)-m(0)<E<U(0)+m(0)$. В этом случае присутствует одна точка поворота $r_{-}$, для которой $v_{-}\left(r_{-}\right)=E-U\left(r_{-}\right)-m\left(r_{-}\right)=0$; при $r>r_{-}$эффективный потенциал положителен и волновая функция осциллирует, в классически запрещенной области $r<r_{-}$эффективный потенциал меньше нуля и волновая функция экспоненциально убывает. 
РЕжим 3: $U(\infty)+m(\infty)<E<U(0)-m(0)$. В этом случае присутствуют две точки поворота $r_{+}<r_{-}$такие, что $v_{ \pm}\left(r_{ \pm}\right)=E-U\left(r_{ \pm}\right) \pm m\left(r_{ \pm}\right)=0$; при $r>r_{-}$и при $r<r_{+}$эффективный потенциал положителен и волновая функция осциллирует, при $r_{+}<r<r_{-}$эффективный потенциал отрицателен и мы имеем дело с туннельной волновой функцией. При $r<r_{+}$стационарные состояния имеют дискретный спектр энергий $E_{j}$ : это либо квазистационарные (гамовские) состояния, которые со временем туннелируют и уходят на бесконечность, либо состояния, пришедшие с бесконечности при резонансном (клейновском) туннелировании (аналогичные эффекты описаны в работе [14]).

Главный член для плотности состояний в нуле в этих трех случаях имеет вид (квазиклассические асимптотики волновых функций и вывод нормировочных констант представлены в приложении А)

$$
\begin{array}{ll}
\text { Режим 1: } & \text { LDOS }_{\text {Dir }}(E)=\frac{v_{+}(0)+v_{-}(0)}{4 \pi h^{2}}=\frac{E-U(0)}{2 \pi h^{2}} ; \\
\text { Режим 2: } & \text { LDOS }_{\text {Dir }}(E)=\frac{m(0)}{2 \pi h^{2}} \exp \left\{-\frac{2}{h} \int_{0}^{r_{-}(E)}\left|n_{1}\right| d r\right\} ; \\
\text { РЕжим 3: } & \text { LDOS }_{\text {Dir }}\left(E_{j}^{ \pm}\right)=\frac{\left|v_{ \pm}(0)\right|}{16 \pi h \Delta E_{j}^{ \pm}} \exp \left\{-\frac{2}{h} \int_{r_{+}\left(E_{j}^{ \pm}\right)}^{r_{-}\left(E_{j}^{ \pm}\right)}\left|n_{1}\right| d r\right\} .
\end{array}
$$

В последней формуле $\Delta E_{j}^{ \pm} \equiv E_{j+1}^{ \pm}-E_{j}^{ \pm}$есть расстояние между дискретными уровнями энергии квазистационарных состояний $E_{j}^{ \pm}$, уровни $E_{j}^{+}$соответствуют угловому моменту $l=0$ и уровни $E_{j}^{-}$- моменту $l=-1$ (см. приложение A).

Для больших энергий плотность состояний такая же, как в безмассовом случае, и равна плотности состояний без иглы, уменьшенной на величину $U(0) / 2 \pi h^{2}$, одинаковую для всех энергий $E>U(0)+m(0)$. В запрещенной области (для энергий $E<U(0)+m(0))$, так же, как и в случае уравнения Шредингера, плотность состояний становится экспоненциально малой. Графики плотности состояний представлены на рис. 2.

Таким образом, в случае уравнения Шредингера игла микроскопа искажает плотность состояний только для энергий, меньших величины наведенного потенциала (плотность состояний становится экспоненциально малой), а плотность состояний для больших энергий остается (в главном) неизменной (см. рис. 2). В случае уравнения Дирака игла микроскопа искажает также состояния с большими энергиями: плотность состояний уменьшается на величину $U(0) / 2 \pi h^{2}$ (линейная зависимость плотности состояний от энергии "сдвигается" по оси абсцисс на величину наведенного потенциала). Кроме того, при наличии массы $m$ экспоненциально малой становится плотность состояний с энергиями меньше $U(0)+m$.

\section{4. ЗАКЛЮЧЕНИЕ}

В представленной работе с помощью квазиклассических асимптотик обобщенных собственных функций двумерных радиально-симметричных операторов Шредингера и Дирака исследованы локальные плотности состояний. Эти исследования позволяют оценить и сравнить эффекты влияния иглы туннельного микроскопа на получаемые при сканировании результаты измерений. 


\section{ПРИЛОЖЕНИЕ А}

\section{Квазиклассические асимптотики}

A.1. Нормировка на дельта-функцию. Рассмотрим уравнение Шредингера (2.1). В надбарьерных состояниях асимптотика выражается через функцию Бесселя и при больших аргументах имеет вид

$$
\begin{aligned}
\psi & =C_{\infty}(E) \frac{\sqrt{T(r)}}{\sqrt{r} \sqrt[4]{E-U(r)}} J_{0}\left(\frac{1}{h} T(r)\right)(1+O(h))= \\
& =C_{\infty}(E) \frac{1}{\sqrt{r}} \sin \left(\frac{1}{h} \sqrt{E} r+O(1)\right)(1+O(h)), \quad r \rightarrow \infty .
\end{aligned}
$$

Константа $C_{\infty}(E)$ определяется из условия (2.4) нормировки на дельта-функцию. Следуя процедуре, описанной в книге [7], рассмотрим уравнение для двух близких волновых чисел $k, k_{1}$ (где $E(k)=h^{2} k^{2}$ ) и применим к уравнению для $k_{1}$ комплексное сопряжение, которое обозначим звездочкой:

$$
\psi_{k}^{\prime \prime}+\frac{1}{r} \psi_{k}^{\prime}+\left(k^{2}-\frac{U}{h^{2}}\right) \psi_{k}=0, \quad \psi_{k_{1}}^{* \prime \prime}+\frac{1}{r} \psi_{k_{1}}^{* \prime}+\left(k_{1}^{2}-\frac{U}{h^{2}}\right) \psi_{k_{1}}^{*}=0
$$

Вычитая одно уравнение из другого, получим

$$
\left(k_{1}^{2}-k^{2}\right) r \psi_{k} \psi_{k_{1}}^{*}=\left(r \psi_{k_{1}}^{*} \psi_{k}^{\prime}-r \psi_{k} \psi_{k_{1}}^{* \prime}\right)^{\prime}
$$

так что

$$
\iint_{\mathbb{R}_{x y}^{2}} \psi_{k} \psi_{k_{1}}^{*} d x d y=2 \pi \lim _{R \rightarrow \infty} \int_{0}^{R} \psi_{k} \psi_{k_{1}}^{*} r d r=\left.\lim _{R \rightarrow \infty} \frac{2 \pi R}{k_{1}^{2}-k^{2}}\left(\psi_{k_{1}}^{*} \psi_{k}^{\prime}-\psi_{k} \psi_{k_{1}}^{* \prime}\right)\right|_{r=R} .
$$

Используя асимптотику на бесконечности и учитывая, что $\psi_{k}^{\prime}(0)=0$, получим

$$
\int_{0}^{R} \psi_{k} \psi_{k_{1}}^{*} r d r=\frac{\left|C_{\infty}\right|^{2}}{2\left(k_{1}^{2}-k^{2}\right)}\left(\left(k-k_{1}\right) \sin \left(\left(k_{1}+k\right) R+O(1)\right)+\left(k+k_{1}\right) \sin \left(\left(k_{1}-k\right) R+O(1)\right)\right) .
$$

Рассмотрим слабый предел при $R \rightarrow \infty$ :

$$
\lim _{R \rightarrow \infty} \sin \left(\left(k+k_{1}\right) R\right)=0, \quad \lim _{R \rightarrow \infty} \frac{\sin \left(R\left(k-k_{1}\right)\right)}{k-k_{1}}=\pi \delta\left(k-k_{1}\right) .
$$

В результате окончательно имеем

$$
\lim _{R \rightarrow \infty} 2 \pi \int_{0}^{R} \psi_{k} \psi_{k_{1}}^{*} r d r=\lim _{R \rightarrow \infty} \frac{\pi\left|C_{\infty}\right|^{2}}{k_{1}-k} \sin \left(\left(k_{1}-k\right) r+O(1)\right)=\pi^{2}\left|C_{\infty}\right|^{2} \delta(r),
$$

откуда получаем значение нормировочной постоянной $C_{\infty}=1 / \pi$.

Аналогичные рассуждения справедливы для системы Дирака. Пусть $u=\psi_{1} \sqrt{r}$, $w=\psi_{2} \sqrt{r}$. Рассмотрим систему для волнового числа $k$ и комплексно-сопряженную систему для волнового числа $k_{1}$. Умножим первое и второе уравнения системы 
для $k$ на $-u_{k_{1}}^{*}$ и на $-w_{k_{1}}^{*}$ соответственно, а первое и второе уравнения для $k_{1}-$ на $u_{k}$ и $w_{k}$, после чего сложим четыре полученных равенства. В результате получим

$$
\left(u_{k} u_{k_{1}}^{*}+w_{k} w_{k_{1}}^{*}\right)\left(k_{1}-k\right)=i \frac{\partial}{\partial r}\left(u_{k} w_{k_{1}}^{*}+u_{k_{1}}^{*} w_{k}\right)
$$

На бесконечности справедливы следующие асимптотики:

$$
\begin{aligned}
\frac{u(r)}{\sqrt{r}}=\psi_{1} & =\frac{C}{\sqrt{2} \sqrt{2 \pi h}} \frac{\sqrt{v_{+} T}}{\sqrt{r n_{1}}} J_{0}\left(\frac{T}{h}\right)(1+O(h))= \\
& =\frac{C}{\pi \sqrt{2}} \frac{\sqrt{v_{+}}}{\sqrt{r n_{1}}} \cos \left(\frac{T}{h}-\frac{\pi}{4}\right)(1+O(h)), \\
\frac{w(r)}{\sqrt{r}}=\psi_{2} & =\frac{n_{1}}{v_{+}} \frac{i C}{\sqrt{2} \sqrt{2 \pi h}} \frac{\sqrt{v_{+} T}}{\sqrt{r n_{1}}} J_{1}\left(\frac{T}{h}\right)(1+O(h))= \\
& =\frac{i C}{\pi \sqrt{2}} \frac{\sqrt{n_{1}}}{\sqrt{r v_{+}}} \sin \left(\frac{T}{h}-\frac{\pi}{4}\right)(1+O(h)),
\end{aligned}
$$

причем $T(r) / h=(r / h) \sqrt{E^{2}-m(\infty)^{2}}+O(1)$. Условие нормировки принимает вид

$$
\begin{aligned}
\delta\left(k-k_{1}\right)= & \iint_{\mathbb{R}_{x y}^{2}}\left\langle\Psi_{k}, \Psi_{k_{1}}^{*}\right\rangle d x d y=\iint_{\mathbb{R}_{x y}^{2}}\left(\psi_{1 k} \psi_{1 k_{1}}^{*}+\psi_{2 k} \psi_{2 k_{1}}^{*}\right) d x d y= \\
= & 2 \pi \int_{0}^{\infty}\left(\psi_{1 k} \psi_{1 k_{1}}^{*}+\psi_{2 k} \psi_{2 k_{1}}^{*}\right) r d r=2 \pi i \lim _{r \rightarrow \infty} \frac{u_{k} w_{k_{1}}^{*}+u_{k_{1}}^{*} w_{k}}{k_{1}-k}= \\
= & \lim _{r \rightarrow \infty} \frac{C^{2}}{\pi\left(k_{1}-k\right)}\left\{\sqrt[4]{\frac{E+m_{\infty}}{E-m_{\infty}} \frac{E_{1}-m_{\infty}}{E_{1}+m_{\infty}}} \times\right. \\
& \quad \times \cos \left(\frac{r}{h} \sqrt{E^{2}-m_{\infty}^{2}}+O(1)\right) \sin \left(\frac{r}{h} \sqrt{\left.\frac{E_{1}^{2}-m_{\infty}^{2}}{E_{1}-m_{\infty}}+O(1)\right)-}\right. \\
& \left.\times \cos \left(\frac{r}{h} \sqrt{E_{1}^{2}-m_{\infty}^{2}}+O(1)\right) \sin \left(\frac{r}{h} \sqrt{E^{2}-m_{\infty}^{2}}+O(1)\right)\right\}= \\
= & \frac{2 C^{2}}{2 \pi} \pi \delta\left(k_{1}-k\right)
\end{aligned}
$$

(здесь предел также понимается в смысле слабой сходимости). Таким образом, нормировочная константа $C=1$.

А.2. Асимптотики вблизи точек поворота и в классически запрещенной области. Для полноты изложения приведем асимптотические формулы для уравнения Дирака вблизи точек поворота и в классически запрещенной области $U(\infty)+m(\infty)<E<U(0)-m(0)$. Две точки поворота $r_{+}<r_{-}$определяются из уравнения $v_{ \pm}\left(r_{ \pm}\right)=E-U\left(r_{ \pm}\right) \pm m\left(r_{ \pm}\right)=0$. Положим

$$
c_{2}=\left(U^{\prime}\left(r_{+}\right)-m^{\prime}\left(r_{+}\right)\right)\left(E-U\left(r_{+}\right)-m\left(r_{+}\right)\right)>0 .
$$


Пусть $l=0$, имеем

$$
\psi_{1}= \begin{cases}\frac{1}{2 \sqrt{\pi h}} \frac{\sqrt{v_{+} T}}{\sqrt{n_{1} r}} J_{0}\left(\frac{1}{h} \int_{r_{-}}^{r} n_{1}(r) d r\right), & r>r_{-}, \\ \frac{1}{\sqrt{2 \pi}} \frac{\sqrt{v_{+}\left(r_{-}\right)}}{\sqrt[6]{h c_{1}} \sqrt{r_{-}}} \operatorname{Ai}\left(\left(r_{-}-r\right) \frac{\sqrt[3]{c_{1}}}{h^{2 / 3}}\right), & r-r_{-}=O(h), \\ \frac{1}{2 \sqrt{\pi h}} \frac{\sqrt{v_{+} T}}{\sqrt{n_{1} r}} \exp \left\{-\frac{1}{h} \int_{r_{+}}^{r_{-}}\left|n_{1}\right| d r\right\} J_{0}\left(i \frac{1}{h} T(r)\right), & r_{+}<r<r_{-}, \\ \frac{1}{2 \sqrt{2 \pi}} \frac{\sqrt[6]{h} \sqrt{-U^{\prime}\left(r_{+}\right)+m^{\prime}\left(r_{+}\right)} \times \sqrt[3]{c_{2}} \sqrt{r_{+}}}{\times} \exp \left\{-\frac{1}{h} \int_{r_{+}}^{r_{-}}\left|n_{1}\right| d r\right\} \mathrm{Bi}^{\prime}\left(\left(r-r_{+}\right) \frac{\sqrt[3]{c_{2}}}{h^{2 / 3}}\right), & r-r_{+}=O(h), \\ \left.\frac{1}{4 \sqrt{\pi h}} \frac{\sqrt{\left|v_{+}(r)\right| T(r)}}{\sqrt{n_{1}(r) r}} \times \frac{1}{h} \int_{r_{+}}^{r_{-}}\left|n_{1}\right| d r\right\} J_{0}\left(\frac{1}{h} \int_{0}^{r} n_{1} d r\right), & r<r_{+} .\end{cases}
$$

Пусть $l=-1$, имеем

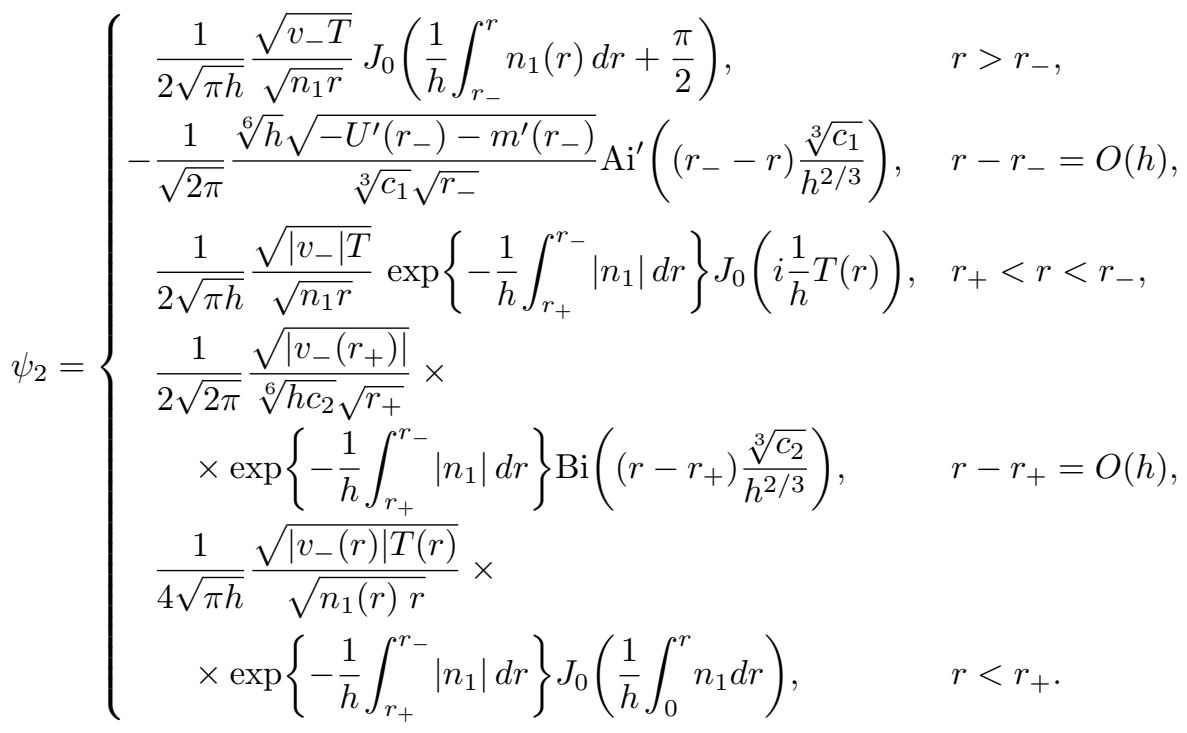

Дискретные уровни энергии $E_{j}^{ \pm}$, соответствующие резонансному туннелированию, определяются из условий квантования:

$$
\begin{array}{ll}
l=0: & \frac{1}{h} \int_{0}^{r_{+}\left(E_{j}^{+}\right)} \sqrt{\left(E_{j}^{+}-U(r)\right)^{2}-m(r)^{2}} d r=2 \pi\left(j+\frac{1}{4}\right), \\
l=-1: & \frac{1}{h} \int_{0}^{r_{+}\left(E_{j}^{-}\right)} \sqrt{\left(E_{j}^{-}-U(r)\right)^{2}-m(r)^{2}} d r=2 \pi j
\end{array}
$$

(здесь $j \in \mathbb{N}$ ). Графики асимптотик представлены на рис. 3. 


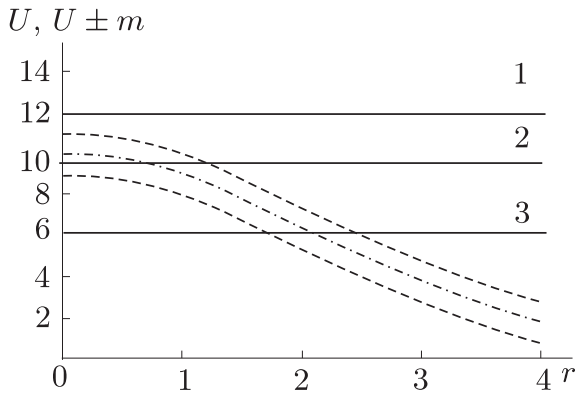

a
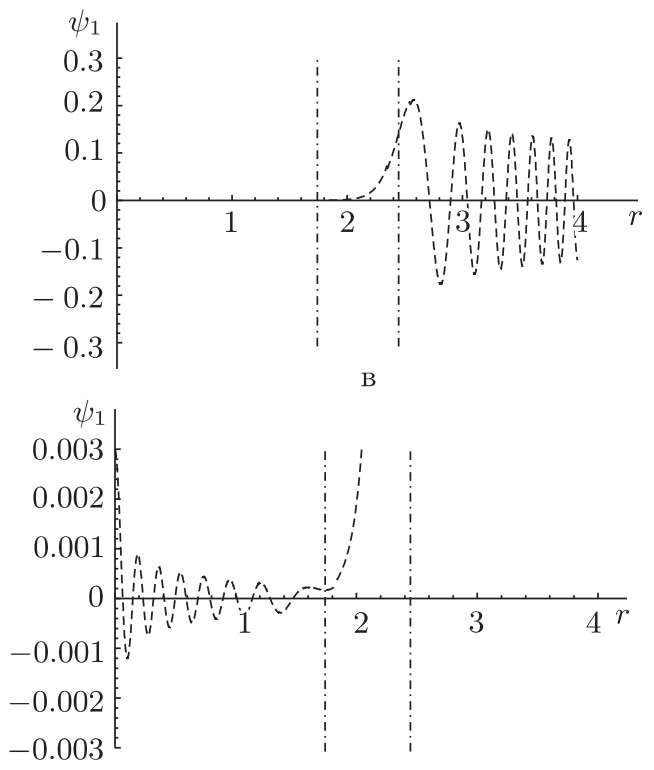

д

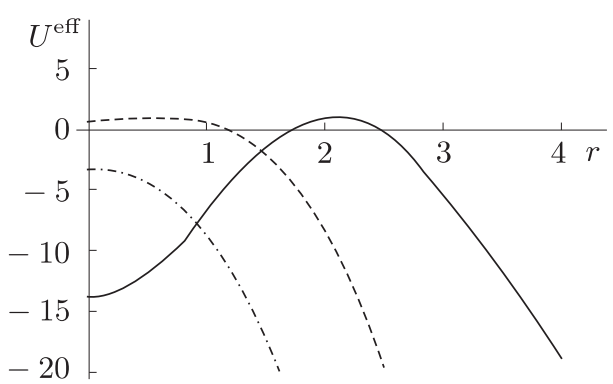

б
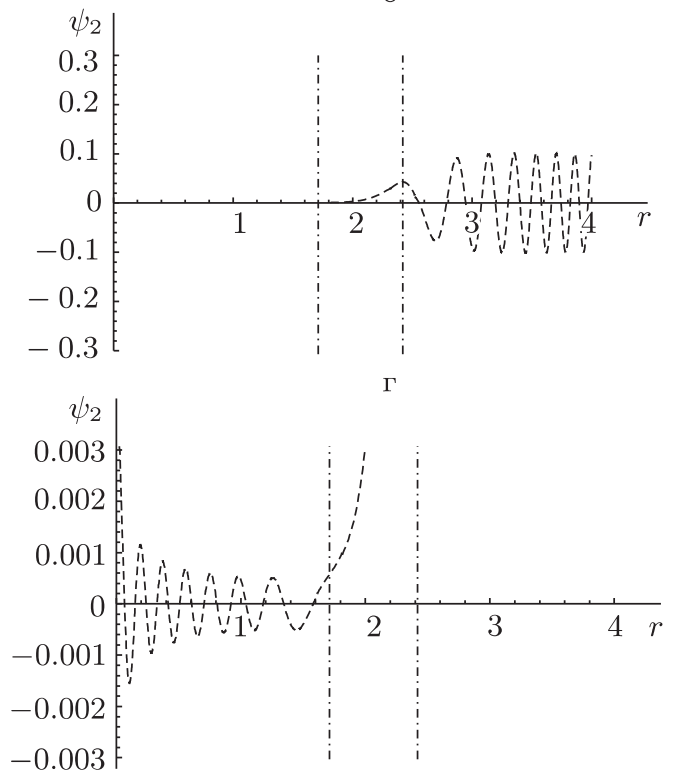

e

Рис. 3. Потенциал $U(r)$ (штрихпунктир) и функции $U(r) \pm m$ (штриховая линия) (а). Эффективный потенциал (б) для случаев $E>U(0)+m(0)$ (штрихпунктирная кривая), $U(0)-m(0)<E<U(0)+m(0)$ (штриховая кривая) и $E<U(0)-m(0)$ (сплошная кривая). Асимптотики для $\psi_{1}(r)$ в разном масштабе (в, д) и асимптотики для $\psi_{2}(r)$ (г, е) в случае 3 . Вертикальные линии на графиках отмечают две точки поворота $r_{ \pm}$, в которых $E-U\left(r_{ \pm}\right) \pm m\left(r_{ \pm}\right)=0$.

\section{ПРИЛОЖЕНИЕ Б}

\section{Точное решение для квадратичного потенциала}

Асимптотики (3.2), (3.3) и (3.5) справедливы при $|E-U(r)| \geqslant \varepsilon>0$. Чтобы исследовать поведение плотности состояний при $E \sim U(0)$, рассмотрим задачу с потенциалом

$$
U(r)=\left\{\begin{array}{cl}
U_{0}-\omega^{2} r^{2}, & r \leqslant r_{0}, \\
0, & r \geqslant r_{0},
\end{array} \quad r_{0}=\frac{\sqrt{U_{0}}}{\omega} .\right.
$$



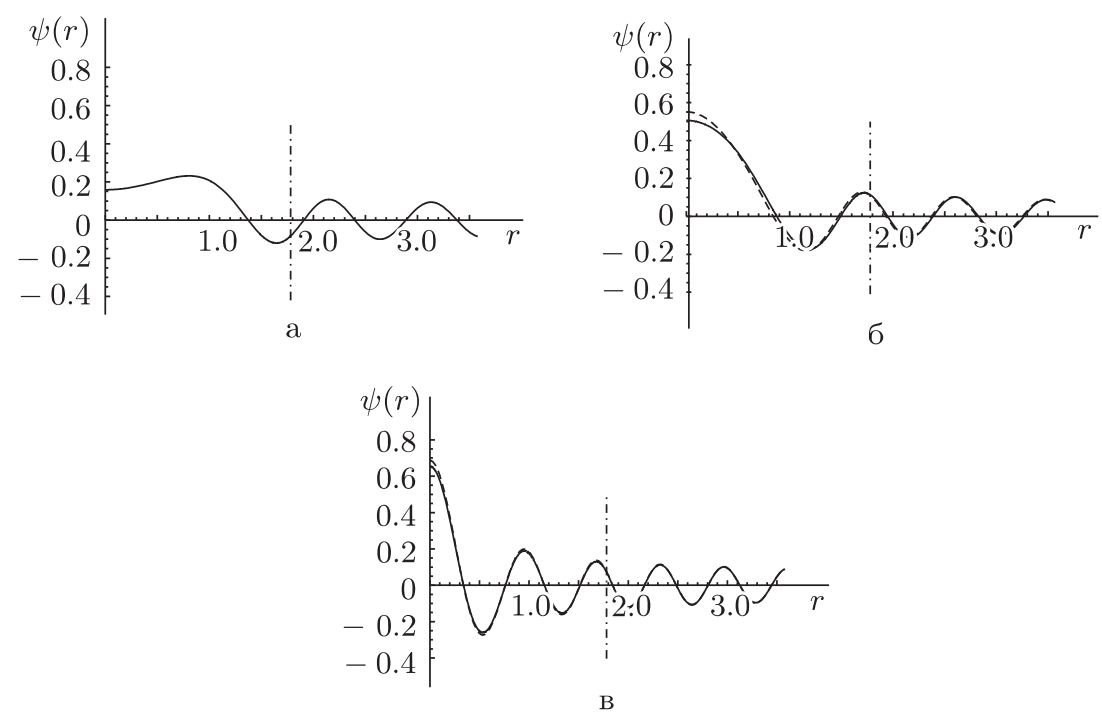

Рис. 4. Точные решения уравнения Шредингера для параболического потенциала (сплошные линии) и асимптотики для потенциала иглы (штриховые линии) при $E-U(0)=-0.05,0.05,0.5$ (а, б, в соответственно).

Тогда уравнение (1.1) примет вид

$$
h^{2} \frac{\partial^{2} \psi}{\partial r^{2}}+h^{2} \frac{1}{r} \frac{\partial \psi}{\partial r}+\left(\left(E-U_{0}\right)+\omega^{2} r^{2}\right) \psi=0
$$

и для него известно точное решение в терминах вырожденной гипергеометрической функции Куммера ${ }^{1)}$ :

$$
\psi(r)= \begin{cases}C e^{(i \omega / 2 h) r^{2}}{ }_{1} F_{1}\left(\frac{1}{2}-i \frac{E-U_{0}}{4 h \omega} ; 1 ;-i \frac{\omega}{h} r^{2}\right), & r \leqslant r_{0}, \\ \frac{\sqrt[4]{E}}{\sqrt{2 \pi h}} J_{0}\left(\frac{1}{h} \sqrt{E} r+\theta_{0}\right), & r \geqslant r_{0},\end{cases}
$$

где константа $C$ и сдвиг фазы $\theta_{0}$ определяются из условия непрерывности решения и его логарифмической производной в точке $r_{0}$ :

$$
\left.\frac{\psi^{\prime}}{\psi}\right|_{r=r_{0}-0}=\left.\frac{\psi^{\prime}}{\psi}\right|_{r=r_{0}+0}, \quad C=\frac{\psi\left(r_{0}+0\right)}{\psi\left(r_{0}-0\right)} .
$$

Решение для $U_{0}=u_{\text {tip }}(0), \omega^{2}=u_{\text {tip }}^{\prime \prime}(0) / 2$ представлено на рис. 4 , плотность состояний для такого потенциала в сравнении с асимптотической плотностью изображена на рис. 2. Расхождение значений точного решения и асимптотики при $r=0$ лежит

1) Вырожденные гипергеометрические функции Куммера определяются как ряд

$$
{ }_{1} F_{1}(a ; b ; z)=\sum_{k=0}^{\infty} \frac{a_{k}}{b_{k}} \frac{z^{k}}{k !}, \quad a_{0}=b_{0}=1, \quad a_{k}=a(a+1) \ldots(a+k-1) .
$$




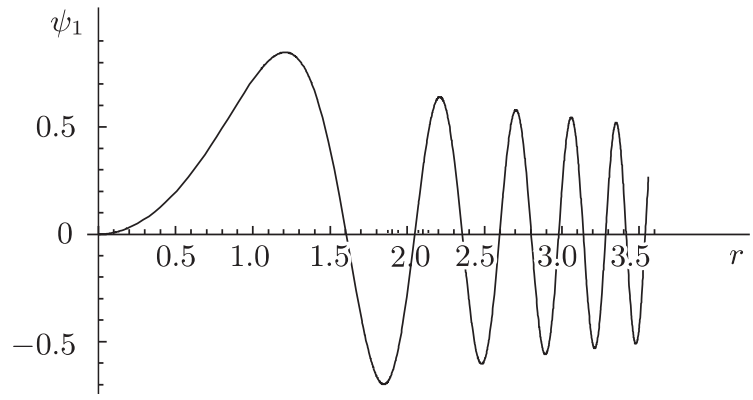

Рис. 5. Точное решение уравнения Дирака при $E=U(0), m \equiv 0$.

в пределах $O(h)$. Расхождение нельзя уменьшить, вычисляя поправки к главному члену, из-за негладкости потенциала, выбранного для точного решения.

Для уравнения Дирака (для эффективного уравнения Шредингера (1.2)) приведем точное решение в случае нулевой массы и квадратичного потенциала при $E=U_{0}$, когда $v(r)=\omega^{2} r^{2}$ при $r<r_{0}$ и $v(r)=E$ при $r \geqslant r_{0}$. В этом случае уравнение принимает вид

$$
h^{2} \frac{\partial^{2}}{\partial r^{2}} \psi_{1}-h^{2} \frac{1}{r} \frac{\partial}{\partial r} \psi_{1}+\omega^{4} r^{4} \psi_{1}=0, \quad r \leqslant r_{0} .
$$

Решение с параболическим потенциалом выражается через функцию Бесселя:

$$
\psi_{1}(r)= \begin{cases}C r J_{1 / 3}\left(\frac{\omega^{2}}{3 h} r^{3}\right), & r \leqslant r_{0}, \\ \frac{1}{\sqrt{2} \sqrt{2 \pi h}} J_{0}\left(\frac{1}{h} E r+\theta_{0}\right), & r \geqslant r_{0} .\end{cases}
$$

Константа $C$ и сдвиг фазы $\theta_{0}$ определяются, как и ранее, из условия непрерывности решения и его логарифмической производной в точке $r_{0}$. Поскольку функция $r J_{1 / 3}(r)$ обращается в нуль при $r=0$, плотность состояний под иглой для $E=U(0)$ равна нулю. Решение изображено на рис. 5.

Благодарности. Авторы признательны В. Е. Назайкинскому и Т. Я. Тудоровскому за ценные дискуссии.

\section{Список литературы}

[1] G. Binnig, H. Rohrer, Ch. Gerber, E. Weibel, Appl. Phys. Lett., 40:2 (1982), 178-179; Phys. Rev. Lett., 49:1 (1982), 57-61; Physica B+C, 109-110:3 (1982), 2075-2077; G. Binnig, H. Rohrer, Helv. Phys. Acta, 55 (1982), 726-735.

[2] J. Tersoff, D. R. Hamann, Phys. Rev. B, 31:2 (1985), 805-813.

[3] V.A. Ukraintsev, Phys. Rev. B, 53:16 (1996), 11176-1185.

[4] J. Brüning, S. Yu. Dobrokhotov, D. S. Minenkov, Russ. J. Math. Phys, 21:1 (2014), 1-8.

[5] T. Mashoff, M. Pratzer, V. Geringer, T. J. Echtermeyer, M. C. Lemme, M. Liebmann, M. Morgenstern, Nano Letters, 10:2 (2010), 461-465, arXiv: 0909.0695.

[6] С.В. Вонсовский, М.И. Кацнельсон, Квантовая физика твердого тела, Наука, М., 1983. 
[7] А. И. Базь, Я. Б. Зельдович, А. М. Переломов, Рассеяние, реакиии и распады в нерелятивистской квантовой механике, Наука, М., 1971.

[8] В. М. Бабич, Матем. сб., 65(107):4 (1964), 576-630.

[9] M. I. Katsnelson, Graphene: Carbon in Two Dimensions, Cambridge Univ. Press, Cambridge, 2012.

[10] В. П. Маслов, Операторные методы, Наука, М., 1973.

[11] В. В. Белов, С. Ю. Доброхотов, Т. Я. Тудоровский, ТМФ, 141:2 (2004), 267-303.

[12] В.П. Маслов, М.В. Федорюк, Квазиклассическое приближение для уравнений квантовой механики, Наука, М., 1976.

[13] С. Ю. Доброхотов, Г.Н. Макракис, В.Е. Назайкинский, Т. Я. Тудоровский, ТМФ, 177:3 (2013), 355-386.

[14] A. V. Shytov, M. I. Katsnelson, L. S. Levitov, Phys. Rev. Lett., 99:24 (2007), 246802, 4 pp., arXiv: 0708.0837.

Поступила в редакцию 10.06.2015 